\title{
Bioinformatics analysis of glial inflammatory responses to air pollution
}

\author{
Chenyu Li, Wei Jiang, Nina Tang and Yan Xu*
}

Dear editor:

We read with great interest the article by Dr. Woodward and colleagues [1], "Toll-like receptor 4 in glial inflammatory responses to air pollution in vitro and in vivo" which appeared in the 15 April 2017 of Journal of Neuroinflammation. Since the results of the article is very attractive for us, we collected original data from NCBI (https:// www.ncbi.nlm.nih.gov/pmc/articles/PMC5391610/bin/129 74_2017_858_MOESM1_ESM.zip) which has been submitted by Woodward et al. and used different methods to perform the bioinformatics analysis in each group; however, we get different results against the article, and we think the author's methods in bioinformatics analysis are inappropriate (Fig. 1).

We noticed that the author used significance analysis microarrays (SAM) in differentially expressed gene (DEG) analysis which, usually, cause high false positives. We utilize a fewer false positives method, Limma (Linear Models for Microarray Analysis) [2] package, which was widely used statistical tests to obtain differential expression based on $\mathrm{R}$ programming language and more accurate than SAM. The results showed 572 RNAs (contain 22 LncRNAs) were differentially expressed between the "control cultures" and "nPM-treated" groups (FDR $<0.05,|\log \mathrm{FC}|>1$ ), and 1931 RNAs (contain 147 LncRNAs) were differentially expressed between the "control cultures" and "LPS-treated" groups (FDR $<0.05$, $|\log \mathrm{FC}|>1$ ) (Additional files 1 and 2). Due to the high false positives, the number of DEGs which were detected by SAM are more than our results. So for high-level analysis, we suggest using Limma or combining more than one method and then only taking the common genes of all methods to get more accurate results [3].

Moreover, for preprocessing microarray data, we recommend using GeneChip Robust Multi-array Averaging (gcRMA). We found that the author used RMA method which results of normalization are very close to gcRMA [4], but gcRMA is an improved algorithm of RMA that can be used to achieve a more accurate expression of the gene chip probes by using sequence-specific probes (Fig. 1). Attached figure shows that the gcRMA is better than RMA in raw data normalization.

\section{Authors' response}

Morgan Levine, Nicholas Woodward and Caleb Finch

Dear Dr. Li,

While we recognize the issues surrounding false discoveries, we do not agree with your comment suggesting "that the methods in bioinformatics analysis [employed in our study] are inappropriate". We are not surprised that your Limma bioinformatics findings differed slightly from SAM in relation to the number of responding genes, which of course varies by method and criteria, because of this reasoning that we tested the robustness of our results using a variety of distinct approaches, rather than relying on any one in particular. Further, our conclusions pointing to the importance of the

* Correspondence: xuyanqyfy@126.com

Department of Nephrology, The Affiliated Hospital of Qingdao University, 16 Jiangsu Road, Qingdao 266003, China
TLR4 pathway are not based on the number of differentially expressed genes-which you imply may be biased-but rather are substantiated by findings derived from state-of-the-art systems biology approaches. For instance, the set of differentially expressed genes based on SAM (which we acknowledge may include false positives) was further analyzed using pathway enrichment and gene ontology analysis. Similarly, our findings were further substantiated using WGCNA-a network analysis approach that identifies higher order modules, rather than individual genes, and thus has been shown to be more robust when it comes to gaining biological insight from high-dimensional data. Finally, and perhaps most importantly, we successfully validated our findings with further experiments both in vitro and in vivo. 

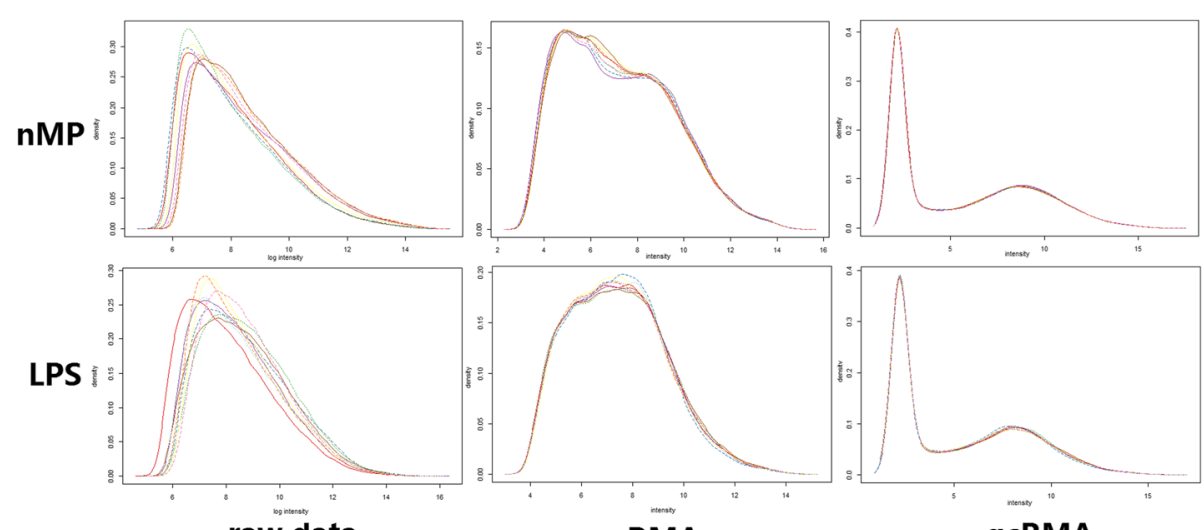

raw data

RMA

gCRMA

Fig. 1 The difference of normalization between RMA and gcRMA

\section{Additional files}

Additional file 1: Differentially expressed RNAs between "LPStreated"and control groups. (CSV $50 \mathrm{~kb}$ )

Additional file 2: Differentially expressed RNAs between " $n P M$ treated"and control groups. (CSV $167 \mathrm{~kb}$ )

\section{Abbreviations}

DEG: Differentially expressed genes; FDR: False discovery rate; gcRMA: GeneChip Robust Multi-array Averaging; Limma: Linear models for microarray analysis; LncRNA: Long non-coding RNA; LPS: Lipopolysaccharide; NCBI: National Center for Biotechnology Information; nPM: Nanoscale particulate matter; RMA: Robust Multi-array Averaging; SAM: Significance analysis microarrays

\section{Funding}

Not applicable.

\section{Availability of data and materials}

Not applicable.

\section{Authors' contributions}

$\mathrm{CL}$ carried out data analysis and drafted the manuscript. WJ provided a deeper insight into the methods of raw data preprocessing and differential gene analysis. NT preformed extra analysis by using RAM and MAS5 methods to preprocess data and using Student's $t$ test, Wilcoxon's signed-rank test, and Moderated T statistic to confirm our results. YX conceived the study, guided the data analysis design, and edited the drafts. All authors read and approved the final manuscript.

\section{Ethics approval}

Not applicable.

\section{Consent for publication}

Not applicable.

\section{Competing interests}

The authors declare that they have no competing interests.

\section{Publisher's Note}

Springer Nature remains neutral with regard to jurisdictional claims in published maps and institutional affiliations.
Received: 5 May 2017 Accepted: 7 August 2017

Published online: 15 August 2017

References

1. Woodward NC, Levine MC, Haghani A, et al. Toll-like receptor 4 in glial inflammatory responses to air pollution in vitro and in vivo. J

Neuroinflammation. 2017;14(1):84

2. Ritchie ME, Phipson B, Wu D, et al. Limma powers differential expression analyses for RNA-sequencing and microarray studies. Nucleic Acids Res. 2015;43(7):e47.

3. Chrominski K, Tkacz M. Comparison of high-level microarray analysis methods in the context of result consistency. PLoS One. 2015;10(6): e0128845.

4. Giorgi FM, Bolger AM, Lohse $M$, et al. Algorithm-driven artifacts in median polish summarization of microarray data. BMC Bioinf. 2010;11:553.
Submit your next manuscript to BioMed Central and we will help you at every step:

- We accept pre-submission inquiries

- Our selector tool helps you to find the most relevant journal

- We provide round the clock customer support

- Convenient online submission

- Thorough peer review

- Inclusion in PubMed and all major indexing services

- Maximum visibility for your research

Submit your manuscript at www.biomedcentral.com/submit
) Biomed Central 